\title{
A novel CSX/NKX2-5 mutation causes autosomal- dominant AV block: are atrial fibrillation and syncopes part of the phenotype?
}

\author{
Ilse Gutierrez-Roelens ${ }^{1}$, Luc De Roy ${ }^{2}$, Caroline Ovaert ${ }^{2}$, Thierry Sluysmans ${ }^{2}$, \\ Koen Devriendt ${ }^{3}$, Han G Brunner ${ }^{4}$ and Miikka Vikkula*,1
}

\begin{abstract}
${ }^{1}$ Laboratory of Human Molecular Genetics, Christian de Duve Institute of Cellular Pathology and Université catholique de Louvain, Brussels, Belgium; ${ }^{2}$ Division of Pediatric Cardiology and Cardiology, Cliniques universitaires St Luc, Université catholique de Louvain, Brussels, Belgium; ${ }^{3}$ Center for Human Genetics, University of Leuven, Leuven, Belgium; ${ }^{4}$ Department of Human Genetics, University Medical Center St Radboud, Nijmegen, The Netherlands
\end{abstract}

The prevalence of congenital heart defects is approximately $1 \%$ of all live births. Identifying the genes responsible for cardiac malformation is the first step to understand pathogenesis. Heterozygous mutations in the CSX/NKX2-5 (NKX2E) gene have been identified to cause atrial septal defect (ASD) and/or atrioventricular (AV) conduction disturbance in some families. However, there is great variability in expressivity of the phenotype between the patients with a CSX/NKX2-5 mutation.

We screened four sporadic patients and three index cases of families with ASD and/or conduction defects. In one of them, a CSX/NKX2-5 mutation was identified. This novel mutation (p.Tyr256X) was inherited in a three-generation family causing five individuals to have cardiac anomalies ranging from ASD to arrhythmias. Interestingly, all the observed AV conduction disturbances were at the nodal level, manifesting first as an AV block of the first degree and evolving toward a second-degree block. Atrial fibrillation, previously reported in three individuals with $C S X / N K X 2-5$ mutations, was observed in three patients.

European Journal of Human Genetics (2006) 14, 1313-1316. doi:10.1038/sj.ejhg.5201702; published online 9 August 2006

Keywords: septal defect; atrial fibrillation; heart; AV block; CSX/NKX2-5

\section{Introduction}

The most frequent phenotype of patients carrying a CSX/ NKX2-5 mutation is atrioventricular (AV) conduction defect (AV block) and secundum atrial septal defect (ASD) (MIM\# 108900). Ventricular septal defect, tetralogy of Fallot, subvalvular aortic stenosis, ventricular hypertrophy, noncompaction of left ventricular myocardium, mitral

${ }^{*}$ Correspondence: Dr M Vikkula, Laboratory of Human Molecular Genetics, Christian de Duve Institute of Cellular Pathology and Université catholique de Louvain, Avenue Hippocrate $74+5$, bp 75.39, Brussels, B-1200, Belgium.

E-mail: vikkula@bchm.ucl.ac.be

Received 15 July 2005; revised 21 April 2006; accepted 14 June 2006; published online 9 August 2006 valve abnormalities, hypoplastic left heart, abnormal systemic venous return and visceral situs inversus have also been reported. ${ }^{1-7}$ Intra- and inter-familial phenotypic variability is noted, but there is no correlation between the mutant alleles and the phenotype. Identification of larger number of mutations and patients would allow more detailed genotype-phenotype comparison.

In human, 29 heterozygous mutations have been identified in CSX/NKX2-5 $5^{1,3-11}$ (MIM\# 600584). Most of them $(62 \%)$ are amino-acid substitutions, and premature STOP codons are identified in the rest of the patients. In addition, a CSX/NKX2-5 screening of 68 formalin-fixed cardiac tissue samples revealed 53 nucleotide changes, but up to 14 nonsynonymous substitutions were identified in a 
single sample. The authors considered them somatic and mosaic in origin. ${ }^{12}$ The disease causative effect needs to be evaluated, as the changes could be artifacts owing to formalin fixation and paraffin embedding.

Two mouse models have helped to understand the molecular basis of the cardiac defects observed in patients carrying CSX/NKX2-5 mutations. ${ }^{13,14}$ In heterozygous Cs $x /$ $N k x 2-5$ knockout (KO) mice, the number of cells in the cardiac conduction system is directly related to Csx/Nkx2-5 gene dosage, and it was stipulated that the specific functional defects associated with Csx/Nkx2-5 loss of function can be attributed to hypoplastic development of the relevant structures in the conduction system. Mice homozygous for a ventricular restricted $\mathrm{KO}$ of $\mathrm{Cs} x / \mathrm{Nk} x 2-5$ actually displayed no structural defects, yet had a massive overgrowth of trabecular muscle and progressive complete heart block owing to hypoplastic AV node at birth.

We report a novel CSX/NKX2-5 mutation identified in a three-generation family with inherited ASD and conduction defects. Detailed phenotypic characterization showed that the conduction defect always resides in the AV node. Interestingly, two family members had syncopes and three had atrial fibrillation.

\section{Materials and methods}

Patients enrolled in this study were clinically examined by L D, Th S, C O, H B and K D. A written informed consent was obtained from all adult participants and from parents of minors in accordance with the ethics committee of Faculty of Medicine, Université catholique de Louvain.
Blood samples were collected from the participants. Genomic DNA was extracted with the Puregene ${ }^{\mathrm{TM}}$ Kit (Gentra Systems).

\section{Exonic mutation screening}

Mutation screening was performed for all the individuals for whom DNA was available, by single-stranded conformation polymorphism (SSCP), heteroduplex analysis and direct sequencing. We used the previously described primer sets and conditions. ${ }^{3}$ All amplified fragments were sequenced on Beckman CEQ 2000 capillary sequencer with the CEQ Dye Terminator Cycle Sequencing Quick Start Kit (Analis, Belgium). Sequences were analysed using the Sequencher software (version 4.1.2, Gene Codes Corp., Ann Arbor, MI, USA).

\section{Results \\ Clinical data}

Seven affected individuals, four sporadic and three index cases of families, were analysed in this study. Three sporadic individuals had an ASD secundum in association with an AV block, and the fourth had only syncopes. The index case of one of the families (family AC-90) presented an ASD secundum and a right bundle branch block, two family members had an ASD with normal ECG (Figure 1e). The second index case had an ASD primum without conduction abnormalities. In this family (AC-83), two other individuals presented an ASD secundum, a slightly asymmetric aortic valve without dysfunction and a borderline congenital AV block grade 1 (Figure 1d).
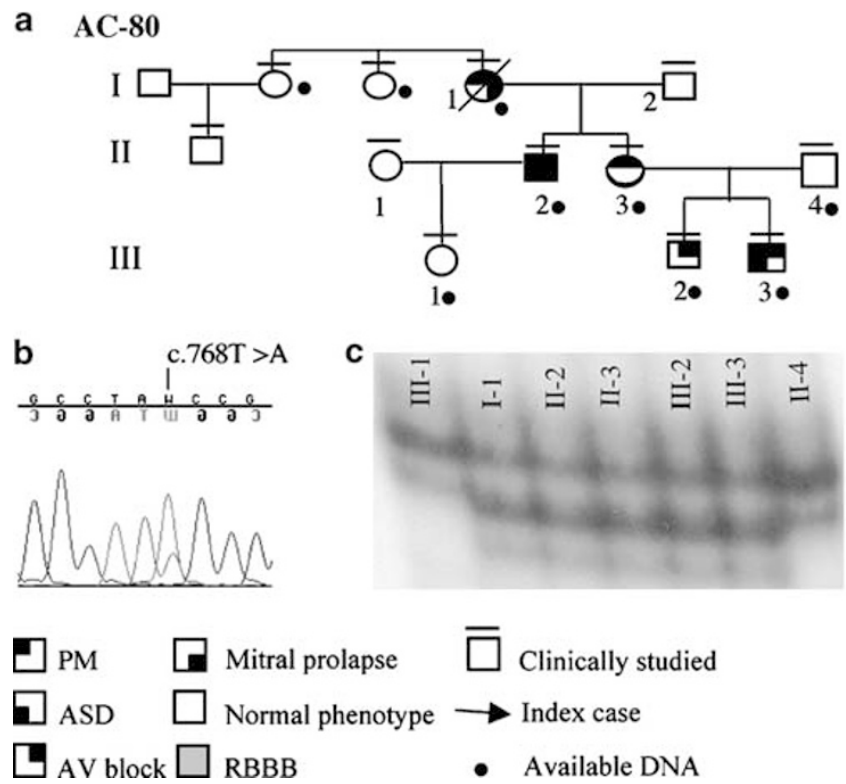

d AC-83
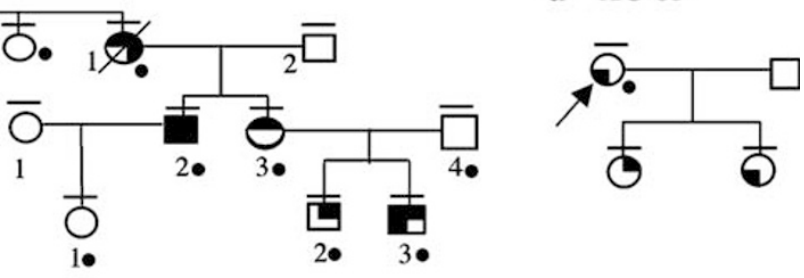

e AC-90

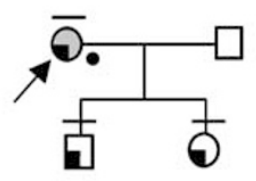

Figure 1 (a) Pedigree drawing for family AC-80. (b) Chromatogram showing the C.768T >A mutation. (c) SSCP results for seven individuals of family AC-80 demonstrate segregation of the c.768T > A mutation. (d) Pedigree drawing for family AC-83. (e) Pedigree drawing for family AC-90. 
In family AC-80, individuals in three generations had cardiac involvement (Figure 1a). Individual I-1 had a second-degree Wenchebach-type AV block and a complete left bundle branch block. A pacemaker was implanted at age 67. In generation II, individual II-2 had a more complex phenotype. He had an atrial ostium secundum septal defect operated at the age of 29 and a mitral valve prolapse. He developed a third-degree AV block, a right bundle branch block and a left anterior fascicular block. A pacemaker was implanted at age 46. Individual II-3 had a second-degree AV block and needed a pacemaker at age 42 . In the third generation, individual III-3 had a small ostium secundum ASD and a first-degree AV block. A pacemaker was implanted at age 15 after several syncopes. Individual III-2, at age 22 years, was an apparently asymptomatic mutation carrier with normal AV conduction $(\mathrm{PR}=166 \mathrm{~ms})$. A prolonged PR (first-degree AV block) was observed during Holter monitoring. Syncopes occurred in at least two individuals (II-3, III-3), although the blocks (second and first degree, respectively) were at the nodal level. III-3 had a marked positive tilt test with high-degree AV block. A complete AV block of $14 \mathrm{~s}$, without any escape rhythm, was provoked during ATP infusion. Interestingly, atrial fibrillation occurred in three members of the family (I-1, II-2 and II-3). An unexplained run of ventricular tachycardia was incidentally discovered once on Holter monitoring in III-3.

\section{Exonic mutation screening}

Exonic mutation screening identified a c.768T $>$ A nucleotide change in the CSX/NKX2-5 coding sequence in family AC-80 (Figure 1b), which creates a premature termination codon, p.Tyr256X. The substitution cosegregates with the disease in the family (Figure 1c). One young individual (III2) carried the mutation and was thought to be asymptomatic. However, Holter monitoring revealed an intermittent first-degree AV block (Figure 1a and c). None of 110 unrelated, unaffected controls carried this nucleotide change. Among the other two familial and four sporadic cases screened, no exonic mutations in $C S X / N K X 2-5$ were identified.

\section{Discussion}

We have identified a novel p. Tyr256X CSX/NKX2-5 mutation in a three-generation family (AC-80) with AV conduction disturbances. Eleven truncating mutations have previously been reported, ${ }^{1,4,6,7,11,15}$ including a deletion of amino acids 198-324 (called M198), which occurs carboxy-terminal to the homeodomain, and overlaps with the region deleted in AC-80. The M198 protein showed normal binding and transactivation of a ANF-luciferase reporter, but a decreased ability to form dimers. ${ }^{8}$ The consequences of the deletion of the last 68 amino acids owing to the AC-80 p.Tyr256X mutation may be similar.
The clinical data of family AC-80 underscore the phenotypic heterogeneity of cardiac septal defects, and suggests atrial fibrillation and ventricular arrhythmias to be associated with the CSX/NKX2-5 phenotype. The AV block seems to be confined to the AV node and may lead to severe symptoms under vagal stimulation, despite the apparently benign presentation. This, and the lability of AV node conduction in time, suggests the need for preventive measures, and points to the importance of early identification of individuals with $C S X / N K X 2-5$ mutations.

No exonic CSX/NKX2-5 mutations were identified in the other two families with phenotypes similar to AC-80. This can be explained by nondetection of intragenic rearrangements, although one index patient was heterozygous for two exonic polymorphisms, or the presence of another causative gene, as proposed by Megarbane et al. ${ }^{16}$

\section{Acknowledgements}

We are grateful to all the family members and patients for their invaluable contributions. These studies were supported by Interuniversity Attraction Poles initiated by the Belgian Science Policy, network $5 / 25$ and the FNRS (Fonds national de la recherche scientifique) (to $M V$, a 'Maitre de recherche du FNRS') and the National Foundation for Research in Pediatric Cardiology (to ThS.). I Gutierrez-Roelens was supported by a fellowship from FRIA (Fonds pour la formation à la recherche dans l'industrie et dans l'agriculture) and Patrimoine UCL. We thank Ms Anne Van Egeren for her expert technical assistance, and Ms Liliana Niculescu for secretarial help.

\section{References}

1 Schott JJ, Benson DW, Basson CT et al: Congenital heart disease caused by mutations in the transcription factor NKX2-5 [see comments]. Science 1998; 281: 108-111.

2 Benson DW, Sharkey A, Fatkin D et al: Reduced penetrance, variable expressivity, and genetic heterogeneity of familial atrial septal defects. Circulation 1998; 97: 2043-2048.

3 Gutierrez-Roelens I, Sluysmans T, Gewillig M, Devriendt K, Vikkula M: Progressive AV-block and anomalous venous return among cardiac anomalies associated with two novel missense mutations in the CSX/NKX2- 5 gene. Hum Mutat 2002; 20: 75-76.

4 Watanabe Y, Benson DW, Yano S, Akagi T, Yoshino M, Murray JC Two novel frameshift mutations in NKX2.5 result in novel features including visceral inversus and sinus venosus type ASD. J Med Genet 2002; 39: 807-811.

5 Elliott DA, Kirk EP, Yeoh T et al: Cardiac homeobox gene NKX2-5 mutations and congenital heart disease: associations with atrial septal defect and hypoplastic left heart syndrome. J Am Coll Cardiol 2003; 41: 2072-2076.

6 McElhinney DB, Geiger E, Blinder J, Benson DW, Goldmuntz E: NKX2.5 mutations in patients with congenital heart disease. J Am Coll Cardiol 2003; 42: 1650-1655.

7 Sarkozy A, Conti E, Neri C et al: Spectrum of atrial septal defects associated with mutations of NKX2.5 and GATA4 transcription factors. J Med Genet 2005; 42: e16.

8 Kasahara H, Lee B, Schott JJ et al: Loss of function and inhibitory effects of human CSX/NKX2.5 homeoprotein mutations associated with congenital heart disease. J Clin Invest 2000; 106: 299-308.

9 Hosoda T, Komuro I, Shiojima I et al: Familial atrial septal defect and atrioventricular conduction disturbance associated with a point mutation in the cardiac homeobox gene CSX/NKX2-5 in a Japanese patient. Jpn Circ J 1999; 63: 425-426. 
10 Goldmuntz E, Geiger E, Benson DW: NKX2.5 mutations in patients with tetralogy of fallot. Circulation 2001; 104: 2565-2568.

11 Ikeda Y, Hiroi Y, Hosoda $\mathrm{T}$ et al: Novel point mutation in the cardiac transcription factor CSX/NKX2.5 associated with congenital heart disease. Circ J 2002; 66: 561-563.

12 Reamon-Buettner SM, Hecker H, Spanel-Borowski K, Craatz S, Kuenzel E, Borlak J: Novel NKX2-5 mutations in diseased heart tissues of patients with cardiac malformations. Am J Pathol 2004; 164: $2117-2125$.

13 Jay PY, Harris BS, Maguire CT et al: Nkx2-5 mutation causes anatomic hypoplasia of the cardiac conduction system. J Clin Invest 2004; 113: 1130-1137.
14 Pashmforoush M, Lu JT, Chen $\mathrm{H}$ et al: Nkx2-5 pathways and congenital heart disease; loss of ventricular myocyte lineage specification leads to progressive cardiomyopathy and complete heart block. Cell 2004; 117: 373-386.

15 Benson DW, Silberbach GM, Kavanaugh-McHugh A et al: Mutations in the cardiac transcription factor NKX2.5 affect diverse cardiac developmental pathways. J Clin Invest 1999; 104: $1567-1573$

16 Megarbane A, Stephan E, Kassab R et al: Autosomal dominant secundum atrial septal defect with various cardiac and noncardiac defects: a new midline disorder. Am J Med Genet 1999; 83: 193-200. 